\title{
Properties of a periodic ansatz for the coarsening of soliton-lattice
}

\author{
Simon Villain-Guillot \\ Laboratoire Onde et Matière d'Aquitaine, Université Bordeaux I, \\ 351 cours de la Libération 33405 Talence Cedex, France*
}

\begin{abstract}
Soliton lattices are periodic solutions of Ginzburg-Landau equation which can be usefull tools to explore the coarsening process (or Ostwald ripening) which takes place during a Cahn-Hilliard dynamics. They can be used to identify the stationary solutions of the dynamics and how these intermediate states are destroyed by fluctuations.

The coarsening process drives the systems from a stationary solution to the next one which is of period double and of lower energy. Using another family of soliton lattices, this process can be described continuously via a phase field equation.

We present here properties of these two families, including the Fourier series decomposition of the non symetric soliton lattice which we use as building block of our ansatz.
\end{abstract}

*Electronic address: simon.villain-guillot@u-bordeaux1.fr 


\section{INTRODUCTION AND PHYSICAL MOTIVATION}

In thermodynamics, when a homogenous system departs suddenly from equilibrium, the fluctuations around the initial ground state are linearly amplified and the homogenous phase can for example spontaneously separate into two different more stable states. The interfaces which delimit the numerous resulting monophasic domains will interact with each other. The domains of the same state will slowly coalesce, minimizing the overall interfacial energy and either give rise to formation of a complex pattern, or merge into a single interface. It results then in only two well separated domains.

When the system is led into a linearly unstable configuration, this process is initiated via a spinodal decomposition. The leading instability then selects a modulation of the order parameter at a well defined length scale, which grows and, due to non-linearities, saturates.

The resulting pattern is composed of well defined interfaces delimiting domains containing one of the two stable phases. Remarkably, this dynamics conserves the modulation width, and the resulting stationary pattern is of almost the same length scale as the one selected initially $[1,2]$. The dynamics finally ends with a much slower, self-inhibiting process, dominated by the interactions between the interfaces. The different regions of each phase coalesce in the so-called Ostwald ripening where the number of domains diminishes whereas their typical size increases. This dynamics can be described as a self similar process of synchronous fusion and evaporation of domains [3], the asymptotic state being composed of only two domains, one for each phase.

In this article, by spinodal decomposition, we refer to the first stage of the dynamics only, while coarsening will denote the second stage. Hillert[4], Cahn and Hilliard[5] have proposed a model equation describing the segregation for a binary mixture. This model, known as the Cahn-Hilliard equation (C-H later on), belongs to the Model B class in Hohenberg and Halperin's classification [6]. It is a standard model for phase transition with conserved quantities and has applications to phase transition in liquid crystals[7], segregation of granular mixtures in a rotating drum [8], or formation of sand ripples $[9,10]$.

The aim of this article is to present a one dimensional ansatz describing continuously the coalescence process. This ansatz is in the form of a one parameter family of symmetric profiles which interpolates between two stationary states composed of homogeneous domains of length $\lambda / 2$ and $\lambda$. It allows to realize a self similar sequence of coalescence process in 
1D, starting from the regular micro phase separated states issued from the non-linear saturation of the spinodal decomposition dynamics and ending with the single interface which characterize the infinite time, thermodynamic stable state.

The paper is organized as follows: first, we present in part II a brief review on general properties of phase segregations and on the $(\mathrm{C}-\mathrm{H})$ model, mainly to fix the notations. We will reproduce briefly the original derivation by Cahn and Hilliard, restricting ourselves to the one dimensional case. In part III, we present a family of symmetric solutions of the GinzburgLandau equation which can be used to study the dynamics of spinodal decomposition and to determine all the symmetric stationary state of the $(\mathrm{C}-\mathrm{H})$ dynamics. Then in part IV, we introduce a non-symmetric family of solutions of the (G-L) equation which is used to construct a continuous interpolation between two consecutive symmetric stationary states. In part $\mathrm{V}$, the main original part of this work, we show as an application that this periodic family of solutions can be used as an ansatz which enable to follow continuously each step of the coarsening process. We use the Fourier series associated with these particular profiles as tools to check the hypotheses and to follow along time the dynamics of coarsening. The Fourier coefficients are computed in the appendix, where detailed calculations are presented.

\section{THE CAHN-HILLIARD MODEL}

The Cahn-Hilliard theory is a modified diffusion equation; it is a continuous conservative model for the scalar order parameter $\Psi$, which reads in its dimensionless form:

$$
\frac{\partial \Psi}{\partial t}(\mathbf{r}, t)=\nabla^{2}\left(\frac{\varepsilon_{0}}{2} \Psi+2 \Psi^{3}-\nabla^{2} \Psi\right)
$$

The real order parameter $\Psi$ can correspond to the dimensionless magnetization in Ising ferromagnet, to the fluctuation of density of a fluid around its mean value during a phase

separation or to the concentration in some region around $\mathbf{r}$ of one of the components of a binary solution. $\varepsilon_{0}$ is the dimensionless control parameter of the system ; it is often identified to the reduced temperature $\left(\varepsilon_{0}=\frac{T-T_{c}}{T_{c}}\right.$ where $T_{c}$ is the critical temperature of the phase transition). This equation, first derived by Cahn and Hilliard [5], has also been retrieved by Langer[3] from microscopic considerations. 
The homogeneous stationary solutions for the $(\mathrm{C}-\mathrm{H})$ equation are extrema of the effective Ginzburg-Landau potential $V(\Psi)=\frac{\varepsilon_{0}}{2} \Psi^{2}+\Psi^{4}$ (G-L later on). For positive $\varepsilon_{0}$, there is only one homogenous solution $\Psi=0$ which is linearly stable; for negative $\varepsilon_{0}$, the stationary solution $\Psi=0$ undergoes a pitchfork bifurcation and three stationary solutions exist. $\Psi=0$ is still a stationary solution, but it is now linearly unstable; two other symmetric solutions $\Psi= \pm \frac{\sqrt{-\varepsilon_{0}}}{2}$ are stable and have the same free energy $F=-\varepsilon_{0}^{2} / 32$. Thus, a first order transition can be experienced by quenching the system suddenly from a positive reduced temperature $\varepsilon$ to a negative one. Spinodal decomposition is the resulting dynamics.

The stability of the solution $\Psi=0$ can be studied by linearizing equation (1) around $\Psi=0$ (i.e. neglecting the nonlinear term $\Psi^{3}$ ); considering $\Psi$ as a sum of Fourier modes:

$$
\Psi(\mathbf{r}, t)=\sum_{\mathbf{q}} \Psi_{q} e^{i \mathbf{q} \cdot \mathbf{r}+\sigma t}
$$

where $\Psi_{q}$ is the Fourier coefficient at $t=0$, we obtain for the amplification factor $\sigma(q)$ (or growth rate) :

$$
\sigma(\mathbf{q})=-\left(q^{2}+\frac{\varepsilon_{0}}{2}\right) q^{2}
$$

It shows immediately that $\Psi=0$ is linearly stable for $\varepsilon_{0}>0$ while a band of Fourier modes are unstable for negative $\varepsilon_{0}$, since $\sigma(\mathbf{q})>0$ for $0<q<\sqrt{\left(-\varepsilon_{0} / 2\right)}$. Moreover, the most unstable mode is for $q_{C-H}=\sqrt{-\varepsilon_{0}} / 2$ (with $\sigma_{\max }=\frac{\varepsilon_{0}^{2}}{16}$ ). This wave number of maximum amplification factor will dominate the first stage of the dynamics; in particular, it explains why the modulations appear at length scales close to $\lambda_{C-H}=2 \pi / q_{C-H}$, the associated wave length. Later on, interfaces separating each domain interact through coalescence dynamics, causing $\langle\lambda\rangle$ to change slowly toward higher values $[1,11]$

We will now use known results on non-homogeneous solutions of the (G-L) equation to study both the saturation of the spinodal decomposition and the coalescence. 


\section{STATIONARY STATES OF THE CAHN-HILLIARD DYNAMICS}

\section{A. Symmetric Soliton Lattice Solutions}

For $\varepsilon<0$, there exists a stationary solution of the one dimensional $(\mathrm{C}-\mathrm{H})$ that relies the two homogenous phases $\Psi= \pm \frac{\sqrt{-\varepsilon}}{2}$

$$
\Psi(x)=\frac{\sqrt{\left|\varepsilon_{0}\right|}}{2} \tanh \left(\frac{\sqrt{\left|\varepsilon_{0}\right|} x}{2}\right) .
$$

Such a monotonic solution describes a continuum interface between the two stable homogeneous phases, and corresponds to the thermodynamically stable solution that ends the phase transition dynamics. But this is a particular member of a one parameter family of stationary solutions of the (G-L) equation

$$
\frac{\varepsilon}{2} \Psi+2 \Psi^{3}-\nabla^{2} \Psi=0
$$

These solutions, the so-called soliton-lattice solutions, are :

$$
\Psi_{k, \varepsilon}(x)=k \Delta \operatorname{Sn}\left(\frac{x}{\xi}, k\right) \text { with } \xi=\Delta^{-1}=\sqrt{2 \frac{k^{2}+1}{-\varepsilon}}
$$

where $\operatorname{Sn}(x, k)$ is the Jacobian elliptic function sine-amplitude, or cnoidal mode. This family of solutions is parametrized by $\varepsilon$ and by the modulus $k \in[0,1]$, or "segregation parameter". These solutions describe periodic patterns of periods

$$
\lambda=4 K(k) \xi, \text { where } K(k)=\int_{0}^{\frac{\pi}{2}} \frac{\mathrm{d} t}{\sqrt{1-k^{2} \sin ^{2} t}}=\frac{\lambda}{4 \xi}
$$

is the complete Jacobian elliptic integral of the first kind. Together with $k$, it characterizes the segregation, defined as the ratio between the size of the homogeneous domains, $0.5 \times \lambda$, and the width of the interface separating them, $2 \times \xi$. The equation $(7)$ and the relation $\xi=\Delta^{-1}$, enable to rewrite this family as :

$$
\Psi_{k, \lambda}(x)=\frac{4 K(k) \cdot k}{\lambda} \operatorname{Sn}\left(\frac{4 K(k)}{\lambda} x, k\right) .
$$

This family of profiles (or alternating interfaces) can be obtained exactly as a periodic sum of single solitons and antisolitons[12]

$$
\sum_{n}(-1)^{n} \tanh (\pi s(x-n))=\frac{2 k(s) K(s)}{\pi s} \operatorname{Sn}(x, k) \text { with } s=\frac{K(k)}{K\left(k^{\prime}\right)} \text { and } k^{\prime 2}=1-k^{2}
$$




\section{B. Antsatz for the Spinodal Decomposition Dynamics}

The preceding family of profiles can be used to explore the spinodal decomposition dynamics. It can be associated with a micro phase separation, locally limited by the finite diffusion coefficient. For $k=1, \operatorname{Sn}(x, 1)=\tanh (x)$, we recover the usual single interface solution (4), of width $2 / \sqrt{|\varepsilon|}$; it is associated with a one soliton solution and corresponds to a strong, or macroscopic segregation. Note that $K(1)$ diverges ; the solution

$$
\Psi_{1, \varepsilon}(x)=\frac{\sqrt{|\varepsilon|}}{2} \tanh \left(\frac{\sqrt{|\varepsilon|}}{2} x\right) .
$$

is thus the limit of infinite $s$, when the solitons, entering in relation (9), are far apart one each others. In the opposite limit (weak segregation regime), it describes a sinusoidal modulation

$$
\lim _{k \rightarrow 0} \Psi_{k, \varepsilon}(x)=k \sqrt{\frac{|\varepsilon|}{2}} \sin \left(\sqrt{\frac{|\varepsilon|}{2}} x\right)=k \frac{2 \pi}{\lambda} \sin \left(\frac{2 \pi}{\lambda} x\right)=k q \sin (q x)
$$

It will correspond to the Fourier mode $q=\frac{2 \pi}{\lambda}$ of the initial white noise, with an arbitrary small amplitude $\nu=k q$. Since initially, the spatial period of the pattern is constant during spinodal decomposition, we identify $\lambda$ with the most unstable wave length obtained with the Cahn Hilliard linear approach, $\lambda=\lambda_{C-H}=\frac{4 \pi}{\sqrt{-\varepsilon_{0}}}$, where $\varepsilon_{0}$ is the quench temperature. Thus, we obtain a one parameter family of profiles $\Psi^{*}(x, k)=\Psi_{k, \lambda_{C-H}}(x)$ which describe very well both the linear growth and the saturation. The dynamics is now reduced to the time evolution of the single free parameter : $k(t)$. Using equations (6) and (7), we find that $\lambda, k$ and $\varepsilon$ are related one to another through the state equation

$$
\varepsilon(k)=-2 \varepsilon_{0}\left(1+k^{2}\right)\left(\frac{4 K(k)}{\lambda}\right)^{2} .
$$

So, this implicit equation tells us that if we fix $\lambda=\lambda_{C-H}$, the dynamics can be reduced to the evolution of $\varepsilon(k(t))$.

Given a periodic function $\Phi$ (obtained from experimental data or numerical simulation of equation (5)) at time $t$, the ansatz assumes that it corresponds to a soliton lattice of the same period: i.e., there exists $k(t)$ such that $\Psi(x, t) \sim \Psi_{k(t), \lambda_{C-H}}(x)$ for each time $t$. The value of $k(t)$ can be determined taking advantage of the general properties of the family of solutions $\Psi^{*}(x, k)$ : either, $k$ can be deduced both from the amplitude of the oscillation equals to $4 k K(k) / \lambda$, or from the relation $k=1-\left((\Psi(\lambda / 2, k) / \Psi(\lambda / 4, k))^{2}-1\right)^{2}$; thirdly, a straightforward computation relates $k$ to the ratio of the two first Fourier modes of the 
soliton lattice $\Psi^{*}(x, k)$. We have observed numerically that the three methods show in general similar results within an error of one percent.

In this approach, $\varepsilon(k(t))$ can be then interpreted as a fictitious temperature or "local temperature" of the domains: it is the temperature extracted from the profile $k(t)$ at a given time, using the correspondence between $\varepsilon$ and $k$ of equation (12). For instance, at $t=0$, the amplitude is small and we find that $k(0)=\frac{\nu \lambda_{m}}{2 \pi} \rightarrow 0$ and thus $\varepsilon(0)=8 \pi^{2} / \lambda^{2} \varepsilon_{0}$, different a priori from $\varepsilon_{0}\left(\varepsilon(0)=\frac{\varepsilon_{0}}{2}\right.$ for $\left.\lambda=\lambda_{C-H}\right)$.

Somehow, the dynamics of $(\mathrm{C}-\mathrm{H})$ can be projected at first order onto a dynamics along the sub-family $\Psi^{*}(x, k)=\Psi_{k, \lambda_{C-H}}(x)$, which can be considered as an attractor of the solutions, i.e. the density profile of the system will evolve with time, staying always close to a function $\Psi^{*}(x, k)$. We can now write Cahn-Hilliard dynamics as

$$
\frac{\partial \Psi}{\partial t}(x, t)=\frac{\partial \Psi^{*}}{\partial k} \cdot \frac{\mathrm{d} k}{\mathrm{~d} t}=\frac{\partial^{2}}{\partial x^{2}}\left(\frac{\varepsilon_{0}}{2} \Psi^{*}+2 \Psi^{* 3}-\nabla^{2} \Psi^{*}\right)=\frac{\varepsilon_{0}-\varepsilon(k(t))}{2} \frac{\partial^{2}}{\partial x^{2}} \Psi^{*}(x, k)
$$

Using a solubility condition, it is possible to solve this equation for $k(t)$ and thus to compute the full nonlinear part of this dynamics, the saturation of the spinodal decomposition, which leads the system in a well defined stationary state [2].

\section{Saturations of the Spinodal Decomposition Dynamics}

According to the previous interpretation of the parameter $\varepsilon$, as $\varepsilon(t=0)=\frac{\varepsilon_{0}}{2}$, the system is initially out of equilibrium. The dynamics will saturate when this fictitious temperature will reach the real thermodynamic one, i.e. the quench temperature $\varepsilon_{0}$. Using equation of state (12) for $\lambda=\lambda_{C-H}$, the dynamics will saturate when $k=k_{0}^{s}$ is solution of the implicit equation:

$$
2\left(1+k_{0}^{s 2}\right) K\left(k_{0}^{s}\right)^{2}=-\frac{\varepsilon_{0} \lambda_{C-H}^{2}}{16}=\pi^{2} \quad \text { that is } \quad k_{0}^{s}=0.687
$$

Note that in this case, the width of the interface, which was initially, just after the quench,

proportional to $\frac{2}{\sqrt{-\varepsilon_{0}}}$ has now become proportional to $\frac{\pi}{\sqrt{-\varepsilon_{0}} K\left(k_{0}^{s}\right)}=\frac{\sqrt{2\left(1+k_{0}^{s 2}\right)}}{\sqrt{-\varepsilon_{0}}} \simeq \frac{1.7}{\sqrt{-\varepsilon_{0}}}$ : the segregation has slightly increased. Langer has shown that the profile thus obtained, $\Psi^{*}\left(x, k_{0}^{s}\right)=\Psi\left(x, k_{0}^{s}, \lambda_{C-H}\right)$, is linearly unstable and he has identified the most unstable mode as an "antiferro" mode, leading to a period doubling. The result of this destabilization is another profile of alternate interface, where the length of the domains is now : 
$\lambda=2 \lambda_{C-H}=\frac{8 \pi}{\sqrt{-\varepsilon_{0}}}$. This means that the new stationary profile is given by $\Psi\left(x, k_{1}^{s}, 2 \lambda_{C-H}\right)$, where $k_{1}^{s}$ is solution of the implicit relation

$$
2\left(1+k_{1}^{s 2}\right) K\left(k_{1}^{s}\right)^{2}=-\frac{\varepsilon_{0}\left(2 \lambda_{C-H}\right)^{2}}{16}=4 \pi^{2}=8\left(1+k_{0}^{s 2}\right) K\left(k_{0}^{s}\right)^{2} \quad \text { that is } \quad k_{1}^{s}=0.985
$$

The interface of this new profile is relatively sharper compared to the size of the homogeneous domains : the width of the interface has increased and is now proportional to $\frac{2 \pi}{\sqrt{-\varepsilon_{0}} K\left(k_{1}^{s}\right)}=$ $\frac{\sqrt{2\left(1+k_{1}^{s 2}\right)}}{\sqrt{-\varepsilon_{0}}} \simeq \frac{2 \times 0.99}{\sqrt{-\varepsilon_{0}}}$ but the size of the homogeneous domains has now double, see Figure (1). Again, this new stationary profile turns out to be linearly unstable with respect to an "antiferro" perturbation of period $4 \lambda_{C-H}$.

Thus these families of profiles and related instabilities enable to describe one dimensional coarsening as a cascade of doubling process, leading continuously from a pattern of wave length $\lambda_{C-H}$ composed of domains separated by interfaces to a single $\tanh \left(\frac{\sqrt{-\varepsilon_{0}}}{2} x\right)$ interface separating two semi infinite domains. Each of these successive intermediate profiles can be described by an element of the above family of soliton lattice $\Psi\left(x, k_{n}^{s}, 2^{n} \times \lambda_{C-H}\right)$. We thus have a family of segregation parameter $\left\{k_{n}^{s}\right\}$, which are determined by the implicit relations

$$
2\left(1+k_{n}^{s 2}\right) K\left(k_{n}^{s}\right)^{2}=-\frac{\varepsilon_{0}\left(2^{n} \lambda_{C-H}\right)^{2}}{16}=\pi^{2} 2^{2 n} .
$$

We have found numerically for the first of them

$$
\begin{array}{|l|l|}
\hline k_{0}^{s}=k^{s}=0.6869795924 & k_{0}^{s} \Delta_{0}^{s}=0.400 \sqrt{-\varepsilon_{0}} \\
\hline k_{1}^{s}=0.9851675587 & k_{1}^{s} \Delta_{1}^{s}=0.496250 \sqrt{-\varepsilon_{0}} \\
\hline k_{2}^{s}=0.99997210165 & k_{2}^{s} \Delta_{2}^{s}=0.499990 \sqrt{-\varepsilon_{0}} \\
\hline k_{3}^{s}=0.9999999999027 & k_{3}^{s} \Delta_{3}^{s}=0.49999846 \sqrt{-\varepsilon_{0}} \\
\hline
\end{array}
$$

$\left\{k_{n}^{s}\right\}$ converges toward $k_{\infty}^{s}=1$ (single interface case) according to the relation :

$$
k_{n}^{\prime 2}=1-k_{n}^{2} \underset{n \rightarrow \infty}{\simeq} 4 \exp (-2 K(k))=4 \exp \left(-\pi 2^{n}\right)
$$

Meanwhile the amplitude of the modulation $k_{n}^{s} \Delta_{n}^{s}$ goes toward $\sqrt{\left|\varepsilon_{0}\right|} / 2$, as can be seen in the second column of the table above. For large $n$, we can conclude from the implicit relation (15) that the ratio of the domain size to the interface width characterized by $K\left(k_{n}^{s}\right)$ behaves as $\pi 2^{n-1}$. Each of the stationary profiles

$\Psi_{n}(x)=\Psi\left(x, k_{n}^{s}, 2^{n} \lambda_{C-H}\right)=\frac{\sqrt{-\varepsilon_{0}} K\left(k_{n}^{s}\right) \cdot k_{n}^{s}}{2^{n} \pi} \operatorname{Sn}\left(\frac{\sqrt{-\varepsilon_{0}} K\left(k_{n}^{s}\right)}{2^{n} \pi} x, k_{n}^{s}\right)=\frac{\sqrt{-\varepsilon_{0}} k_{n}^{s}}{\sqrt{2\left(1+k_{n}^{s 2}\right)}} \operatorname{Sn}\left(\frac{\sqrt{-\varepsilon_{0}}}{\sqrt{2\left(1+k_{n}^{s 2}\right)}} x, k_{n}^{s}\right)$ 
for which the interface width is proportional to $\frac{2^{n} \pi}{\sqrt{-\varepsilon_{0}} K\left(k_{n}^{s}\right)}$ (which tends to $\frac{2}{\sqrt{-\varepsilon_{0}}}$, in agreement with $\left.\tanh \left(\frac{\sqrt{-\varepsilon_{0}}}{2} x\right)\right)$, is identically destroyed by the Langer "antiferro" instability.

Note that if we had taken for the initial equation

$$
\frac{\partial \Psi}{\partial t}(\mathbf{r}, t)=\nabla^{2}\left(\alpha \Psi+\beta \Psi^{3}-\nabla^{2} \Psi\right)
$$

then we would have found for the amplification factor $\sigma(q)$ :

$$
\sigma(\mathbf{q})=-\left(q^{2}+\alpha\right) q^{2}
$$

for which the most unstable mode is $q_{C-H}=\sqrt{-\alpha / 2}$ and $\lambda_{C-H}=2 \sqrt{2} \pi / \sqrt{-\alpha}$. Meanwhile, the period of the static Ginzburg-Langau solution would be

$$
\xi=2 \beta^{-1} \Delta^{-1}=\sqrt{\frac{k^{2}+1}{-\alpha}}
$$

leading to the same implicit relation and therefore to the same family of modulus $\left\{k_{n}^{s}\right\}$

$$
\begin{aligned}
\lambda=\lambda_{C-H} & =4 K(k) \xi \\
2 \sqrt{2} \pi / \sqrt{-\alpha} & =4 K(k) \sqrt{\frac{k^{2}+1}{-\alpha}} \\
\sqrt{2\left(k^{2}+1\right)} K(k) & =\pi
\end{aligned}
$$

\section{AN ANSATZ FOR THE 1D COARSENING PROCESS}

\section{A. Non-symmetric soliton lattice Profile}

In order to describe one step of the coalescence process, i.e. the dynamics that start from $\Psi_{n}(x)$ and ends with the profile $\Psi_{n+1}(x)$, we will use another family of equilibrium profiles[13], solutions of (G-L) equation, which write:

$$
\psi(a, k, x)=\frac{\alpha(a, k)-k / \sqrt{a} \beta(a, k) \operatorname{Sn}\left(2 x \frac{K(k)}{\lambda}, k\right)}{1-k / \sqrt{a} \operatorname{Sn}\left(2 x \frac{K(k)}{\lambda}, k\right)}
$$

where $\alpha(a, k)=\frac{-2 k^{2} / a+1+k^{2}}{\left(\left(1+k^{2}\right)^{2}-12 k^{2}+2\left(a+k^{2} / a\right)\left(1+k^{2}\right)\right)^{\frac{1}{2}}}$ and $\beta(a, k)=\frac{2 a-1-k^{2}}{\left(\left(1+k^{2}\right)^{2}-12 k^{2}+2\left(a+k^{2} / a\right)\left(1+k^{2}\right)\right)^{\frac{1}{2}}}$.

It is still a periodic lattice of interfaces, of period $2 \lambda$, but now, the mean value of the order parameter is non zero (non symmetric case). It is controlled by the parameter $a \geqslant 1$ : if $a$ goes infinity, we recover the previous family of periodic profiles. 


\section{B. Ansatz for the continuous interpolation between two stationary states}

If we choose $a$ to be equal to $1+k^{\prime}$ (where $k^{\prime 2}=1-k^{2}$ ), we can then construct symmetric profiles using the sum of two non-symmetric ones. Indeed, using Gauss' transformation (or descending Landen transformation [14]), which relates the soliton lattice of spatial period $2 \lambda$ and modulus $k$ to the soliton lattice of period $\lambda$ and $\operatorname{modulus} \mu=\operatorname{Gauss}(k)=\frac{1-k^{\prime}}{1+k^{\prime}}$, we have

$$
\begin{aligned}
& 1-\frac{\sqrt{5-k^{2}}}{2}\left(\psi\left(k, x-\frac{\lambda}{4}\right)+\psi\left(k, x+\frac{\lambda}{4}\right)\right)=k \operatorname{Sn}\left(2 x \frac{K(k)}{\lambda}, k\right) \\
& 1-\frac{\sqrt{5-k^{2}}}{2}\left(\psi\left(k, x-\frac{\lambda}{2}\right)+\psi\left(k, x+\frac{\lambda}{2}\right)\right)=\left(1-k^{\prime}\right) \operatorname{Sn}\left((4 x+\lambda) \frac{K(\mu)}{\lambda}, \mu\right)
\end{aligned}
$$

with the notation $\psi(k, x)=\psi\left(a=1+k^{\prime}, k, x\right)$. Thus, we then can show from equation $(21)$ that

$$
\frac{K(k)}{\lambda}\left[2-\sqrt{5-k^{2}}\left(\psi\left(k, x-\frac{\lambda}{4}\right)+\psi\left(k, x+\frac{\lambda}{4}\right)\right)\right]=\frac{4 k K(k)}{2 \lambda} \operatorname{Sn}\left(4 x \frac{K(k)}{2 \lambda}, k\right) .
$$

This is the solution of the G-L equation of period $2 \lambda$. Moreover, using the properties of Landen Transformation, we get :

$$
\left(1-k^{\prime}\right) K(k)=2 \mu K(\mu)
$$

and using relation (22), the solution of the (G-L) equation of period $\lambda$ can be expressed as

$$
\frac{K(k)}{\lambda}\left[2-\sqrt{5-k^{2}}\left(\psi\left(k, x-\frac{\lambda}{2}\right)+\psi\left(k, x+\frac{\lambda}{2}\right)\right)\right]=\frac{4 \mu K(\mu)}{\lambda} \operatorname{Sn}\left((4 x+\lambda) \frac{K(\mu)}{\lambda}, \mu\right) .
$$

So, we see that both the initial state $\Psi^{*}(x, \mu, \lambda)=\Psi^{*}\left(x, k_{n-1}^{s}, 2^{n-1} \lambda_{C-H}\right)$ and the final state $\Psi^{*}(x, k, 2 \lambda)=\Psi^{*}\left(x, k_{n}^{s}, 2^{n} \lambda_{C-H}\right)$ of a step of the coalescence process can be describe, modulo a phase shift, by the same function :

$$
\Phi(x, k, \phi)=\frac{K(k)}{\lambda}\left[2-\sqrt{5-k^{2}}\left(\psi\left(k, x-(1-\phi / 2) \frac{\lambda}{2}\right)+\psi\left(k, x+(1-\phi / 2) \frac{\lambda}{2}\right)\right)\right]
$$

with $k=k_{n}^{s}, k_{n-1}^{s}=\operatorname{Gauss}\left(k_{n}^{s}\right)$ and $\lambda=2^{n-1} \lambda_{C-H}$. Therefore we can describe the coalescence by a transformation at constant segregation parameter $k$, during which the degree of freedom $\phi$, associated with the relative phase between the two profiles, evolves in time from 0 to 1 according to the $\mathrm{C}-\mathrm{H}$ dynamics.

This non up-down-symmetric lattice of interfaces can be interpreted as a periodic sum of alternating single interfaces (kinks and antikinks). In the same spirit as relation (9), if one forgets in the infinite sum every two out of four interfaces, one gets :

$$
\psi(x) \backsim \sum_{p}[\tanh (\pi s(x-4 \times p))-\tanh (\pi s(x-4 \times p+1))] .
$$


Then (see Figure (2)) adding $\psi(x+2)$ to $\psi(x)$ enables to recover relation (9), while, after a translation, adding $\psi(x+1)$ and $\psi(x)$ gives the soliton lattice of double period, because of the cancellation of half of the interfaces (annihilation of kinks and antikinks).

The relation (26) can also be written in a more symmetric ways

$$
\Phi(x, k, \phi)=\frac{K(k)}{\lambda}\left[\widehat{\Psi}\left(k, x-(1-\phi / 2) \frac{\lambda}{2}\right)+\widehat{\Psi}\left(k, x+(1-\phi / 2) \frac{\lambda}{2}\right)\right]
$$

where

$\widehat{\Psi}(k, x)=1-\sqrt{5-k^{2}} \psi(k, x)=\frac{k \sqrt{a} \operatorname{Sn}\left(2 x \frac{K(k)}{\lambda}, k\right)-k^{2} / a}{1-k / \sqrt{a} \operatorname{Sn}\left(2 x \frac{K(k)}{\lambda}, k\right)}=\frac{1-k^{\prime}-\left(1+k^{\prime}\right) \sqrt{1-k^{\prime}} \operatorname{Sn}\left(2 x \frac{K(k)}{\lambda}, k\right)}{\sqrt{1-k^{\prime}} \operatorname{Sn}\left(2 x \frac{K(k)}{\lambda}, k\right)-1}$

is still a non up-down-symmetric function but of zero mean values : $\int_{0}^{\lambda} \widehat{\Psi}(k, x)=0$

If we look at the time evolution of the profile $\Phi(x, k, \phi)$, starting from $\phi \simeq 0$, we can transform the $(\mathrm{C}-\mathrm{H})$ equation into a phase field equation, replacing $\frac{\partial}{\partial t} \Phi(x, k, \phi)$ by $\frac{\partial}{\partial \phi} \Phi(x, k, \phi(t)) \times \frac{d \phi}{d t}$. The dynamics will be similar to a spinodal decomposition, with $\phi$ growing and saturating exponentially. $\frac{\partial}{\partial \phi} \Phi(x, k, \phi)$ is the most unstable mode found in Langer's linear stability analysis, characterized by the alternate growth and decrease of domains ("antiferro" mode). Note that when Langer was studying the most unstable perturbation, he was looking at the linearized version of $\mathrm{C}-\mathrm{H}$ equation around $\Psi^{*}(k, x)=\Psi\left(x, k, \lambda_{C-H}\right)$ :

$$
\mathcal{L}(\varphi)=\left(\frac{\varepsilon_{0}}{2}+6 \Psi^{* 2}-\nabla^{2}\right) \varphi=\left(\frac{\varepsilon_{0}}{2}+l \times(l+1) \Psi^{* 2}-\nabla^{2}\right) \varphi
$$

$\mathcal{L}(\varphi)=E \varphi$ is the Lamé equation, for $l=2$. This equation doesn't have simple (algebraic) exact eigenfunction of period $2 \lambda_{C-H}[15] \cdot \frac{\partial}{\partial \phi} \Phi(x, k, \phi)$ for $\phi=0$ is not an exact eigenfunction either [16]. Nevertheless, it happens to be a good approximation for the eigenfunction of lowest eigenvalue. Due to the concavity of $\mathcal{F}(\phi)$ around $\phi=0$ (see below Figure (4)), this eingenvalue will be negative, triggering a linear destabilization and an exponential amplification of the perturbation, i.e. an exponential growth of the translation $\phi$ with time.

Langer's phenomenon of "antiferro" instability appears due to the existence of two possible directions for displacement of the interfaces "tanh" (or of the non-symmetric lattice of interfaces $\psi$ ), one with a positive velocity $\left(+\frac{d \phi}{d t}\right)$ and one with a negative one $\left(-\frac{d \phi}{d t}\right)$. The four different kinds of interfaces present in a cell of length $2 \lambda_{C-H}$ have alternately a positive or a negative velocity. This can be seen as the existence of two antisymmetric patterns [17], or building blocks for the leading instability around a intermediate state $\Psi^{*}\left(x, k_{n}^{s}, 2^{n-1} \times \lambda_{C-H}\right)$ 
(see Figure (3)). These two building blocks are $\pm \frac{d}{d x} \psi(x)$ and are associated with the two pairs of interfaces, $\psi(x)$ and $\psi(x+2)$ which have been used to construct our ansatz.

Note that in Langer's analysis, the breaking of symmetry for the choice of the antiferro cell, corresponds here to the freedom we have when choosing the range of variation of $\phi$ : we could have chosen to go from 0 to -1 , ending after a step of coarsening with the symmetric pattern, or equivalently, the pattern translated of half a period.

\section{Energy landscape}

In order to prove the usefulness of this ansatz, we have plot the energy averaged over the final period, $\mathcal{F}(\phi)=\int F(\Phi(x, k, \phi)) \mathrm{d} x$, as a function of the parameter $\phi$, keeping $k$ constant. We see for example in Figure (4) that the value $\phi=0$ correspond to a local maximum of energy, while $\phi=1$ (or -1 ) is a minimum. Note that there is no energy barrier in this particular energy landscape, in agreement with linear stability analysis.

\section{CONCLUSION : FOURIER DECOMPOSITION AND SOME APPLICATIONS}

As $\psi(k, x)$, the building blocks of our ansatz, are $2 \lambda$ periodic, we can decompose them in Fourier series with $w=2 \pi / T=\pi / \lambda$.

$$
\psi(k, x)=\sum a_{n} \cos (n w x)+b_{n} \sin (n w x)
$$

where the Fourier coefficients $a_{n}$ and $b_{n}$ are computed in the Appendix and are such that

$$
\begin{aligned}
\widehat{\Psi}(k, x) & =1-\sqrt{5-k^{2}} \psi(k, x) \\
& =\frac{2 \pi}{K(k)} \sum \frac{q(k)^{n+1 / 2}}{q(k)^{2 n+1}-1}\left[2 \frac{(-1)^{n} q(k)^{n+1 / 2}}{1+q(k)^{2 n+1}} \cos (2 \pi(2 n+1) x / \lambda)+\frac{\sin ((2 n+1) \pi x / \lambda))}{\sin \left(n \frac{\pi}{2}-\frac{\pi}{4}\right)}\right]
\end{aligned}
$$

where $q(k)=\exp \left(-\pi \frac{K\left(k^{\prime}\right)}{K(k)}\right)$ (see equation (30) of the appendix). This enables to write (equation (31) of the appendix)

$$
\begin{gathered}
\Phi(x, k, \phi)=\frac{K(k)}{\lambda}\left[\widehat{\Psi}\left(k, x-(1-\phi / 2) \frac{\lambda}{2}\right)+\widehat{\Psi}\left(k, x+(1-\phi / 2) \frac{\lambda}{2}\right)\right] \\
=\frac{4 \pi}{\lambda} \sum \frac{(-1)^{n} q(k)^{n+1 / 2}}{1-q(k)^{2 n+1}}\left[2 \frac{q(k)^{n+1 / 2}}{1+q(k)^{2 n+1}} \cos \left((4 n+2) \frac{\pi x}{\lambda}\right) \cos (\pi(2 n+1) \phi / 2)+\frac{\sin ((2 n+1) \pi x / \lambda) \sin \left(\left(n \pi / 2+\frac{\pi}{4}\right) \phi\right)}{\cos \left(n \frac{\pi}{2}+\frac{\pi}{4}\right)}\right]
\end{gathered}
$$


This last result can be useful to track the dynamics of the coarsening. It enable to relate the evolution of the profile in some specific point. For example, in $x=0$, the profile is directly related to phase parameter $\phi$ (which evolves in time), and to the segregation parameter (which should remain constant according to our hypothesis) :

$$
\begin{aligned}
\Phi(0, k, \phi) & =\frac{8 \pi}{\lambda} \sum \frac{q(k)^{n+1 / 2}}{1-q(k)^{2 n+1}} \frac{(-1)^{n} q(k)^{n+1 / 2}}{1+q(k)^{2 n+1}} \cos (\pi(2 n+1) \phi / 2) \\
& =\frac{4 \mu K(\mu)}{\lambda} \operatorname{Cd}(K(\mu) \phi, \mu)=\frac{4 \mu K(\mu)}{\lambda} \operatorname{Sn}(K(\mu)(\phi+1), \mu)
\end{aligned}
$$

This is also true for the profile in $x=\lambda / 4$ and $x=-\lambda / 4$ :

$$
\begin{aligned}
\Phi(\lambda / 4, k, \phi) & =-\Phi(-\lambda / 4, k, \phi)=\frac{4 \pi}{\lambda} \sum(-1)^{n} \frac{q(k)^{n+1 / 2}}{1-q(k)^{2 n+1}} \tan ((2 n+1) \pi / 4) \sin \left(\left(n \pi / 2+\frac{\pi}{4}\right) \phi\right) \\
& =\frac{4 \pi}{\lambda} \sum \frac{q(k)^{n+1 / 2}}{1-q(k)^{2 n+1}} \sin \left(\left(n \pi / 2+\frac{\pi}{4}\right) \phi\right)=2 k K / \lambda \operatorname{Sn}(K \phi / 2)
\end{aligned}
$$

The (adiabatic) hypothesis underlying our approach postulates that coalescence is a dynamics at constant segregation parameter $k$; the sole degree of freedom varying in time is the relative phase between the two profiles $\phi$. To check the hypothesis, for example numerically, one can use the values of the profile in $x= \pm \lambda / 2$ where the amplitude of the profile varies the most (less) as it corresponds to the center of the domain which disappears (grows). In these two points

$$
\begin{aligned}
\Phi(\lambda / 2, k, \phi)+\Phi(-\lambda / 2, k, \phi) & =-\frac{16 \pi}{\lambda} \sum \frac{(-1)^{n} q(k)^{2 n+12}}{1+q(k)^{2(2 n+1)}} \cos (\pi(2 n+1) \phi / 2) \\
& =-\frac{8 \mu K(\mu)}{\lambda} \operatorname{Cd}(K(\mu) \phi, \mu)=-2 \Phi(0, k, \phi)
\end{aligned}
$$

This is a strong constraint on the dynamics that should be verified if the dynamics can be approximate on this particular family of coarsening soliton lattice ; it relates the amplitudes in $x= \pm \lambda / 2$ to the amplitude in $x=0$, and is indeed almost verified numerically.

We also have the useful relation :

$$
\begin{aligned}
\Phi(\lambda / 2, k, \phi)-\Phi(-\lambda / 2, k, \phi) & =4 k K / \lambda\left(\operatorname{Sn}\left(\frac{K(k)}{2}(\phi+1)\right)+\operatorname{Sn}\left(\frac{K(k)}{2}(\phi-1)\right)\right) \\
& =\frac{k^{\prime}}{\sqrt{1+k^{\prime}}} \frac{8 k K / \lambda \operatorname{Sn}\left(\frac{K(k)}{2} \phi\right)}{1-\left(1-k^{\prime}\right) \operatorname{Sn}^{2}\left(\frac{K(k)}{2} \phi\right)}
\end{aligned}
$$


Using this relation, from numerical or experimental values of a given $2 \lambda$ periodic profile at the two special points $x=\lambda / 2$ and $x=-\lambda / 2$, one can extract $\operatorname{Sn}\left(\phi \frac{K(k)}{2}\right)$ and thus the phase $\phi$ at a given time of the dynamics, like in Figure (4).

When investigating a coarsening process, theses results enable to map a profile of the order parameter onto a soliton lattice solution. This particular family of coarsening profiles can therefore be a useful tool to investigate in various 1D systems Ostwald ripening dynamics and its interruption which might lead to pattern formations [18].

\section{Appendix : Fourier decomposition}

As $\psi(k, x)$, the building blocks of our ansatz, are $2 \lambda$ periodic, we can decompose them in Fourier series with $w=2 \pi / T=\pi / \lambda$.

$$
\psi(k, x)=\sum a_{n} \cos (n w x)+b_{n} \sin (n w x)
$$

Using the Fourier series for the cnoidal function of period $2 \lambda$ and $q(k)=\exp \left(-\pi \frac{K\left(k^{\prime}\right)}{K(k)}\right)$, we can write

$$
\begin{gathered}
k \operatorname{Sn}\left(4 x \frac{K(k)}{2 \lambda}, k\right)=\frac{2 \pi}{K(k)} \sum \frac{q(k)^{n+1 / 2}}{1-q(k)^{2 n+1}} \sin ((2 n+1) \pi x / \lambda)=\left[1-\frac{\sqrt{5-k^{2}}}{2}\left(\psi\left(k, x-\frac{\lambda}{4}\right)+\psi\left(k, x+\frac{\lambda}{4}\right)\right)\right] \\
=1-\frac{\sqrt{5-k^{2}}}{2} \sum a_{p}\left[\cos \left(p w\left(x-\frac{\lambda}{4}\right)\right)+\cos \left(p w\left(x+\frac{\lambda}{4}\right)\right)\right]+b_{p}\left[\sin \left(p w\left(x-\frac{\lambda}{4}\right)\right)+\sin \left(p w\left(x+\frac{\lambda}{4}\right)\right)\right] \\
=1-\sqrt{5-k^{2}} \sum\left(a_{p} \cos (p w x) \cos \left(p w \frac{\lambda}{4}\right)+b_{p} \sin (p w x) \cos \left(p w \frac{\lambda}{4}\right)\right) \\
=1-\sqrt{5-k^{2}} \cos \left(p \frac{\pi}{4}\right) \sum\left(a_{p} \cos (p \pi x / \lambda)+b_{p} \sin (p \pi x / \lambda)\right)
\end{gathered}
$$

So when $p$ is such that $\cos \left(p \frac{\pi}{4}\right) \neq 0$ we can conclude that $a_{0}=\left(5-k^{2}\right)^{-\frac{1}{2}}, a_{4 n}=a_{4 n+1}=$ $a_{4 n+3}=0, b_{4 n}=0$ and

$$
b_{2 n+1}=\frac{2 \pi}{K(k) \sqrt{5-k^{2}} \sin \left(n \frac{\pi}{2}-\frac{\pi}{4}\right)} \frac{q(k)^{n+1 / 2}}{1-q(k)^{2 n+1}} .
$$

Using Landen transformation

$$
q(\mu)=\exp \left(-\pi \frac{K\left(\mu^{\prime}\right)}{K(\mu)}\right)=\exp \left(-2 \pi \frac{K\left(k^{\prime}\right)}{K(k)}\right)=q(k)^{2} .
$$


together with $2 \mu K(\mu)=\left(1-k^{\prime}\right) K(k)$, we find similarly

$$
\begin{aligned}
& \left(1-k^{\prime}\right) \operatorname{Sn}\left((4 x+\lambda) \frac{K(\mu)}{\lambda}, \mu\right)=\left[1-\frac{\sqrt{5-k^{2}}}{2}\left(\psi\left(k, x-\frac{\lambda}{2}\right)+\psi\left(k, x+\frac{\lambda}{2}\right)\right)\right] \\
= & \frac{2 \pi\left(1-k^{\prime}\right)}{\mu K(\mu)} \sum_{n=0} \frac{q(\mu)^{n+1 / 2}}{1-q(\mu)^{2 n+1}} \sin \left((2 \mathrm{n}+1) \pi \frac{4 x+\lambda}{2 \lambda}\right)=1-\sqrt{5-k^{2}} \sum \cos \left(p \frac{\pi}{2}\right)\left(a_{p} \cos (p w x)+b_{p} \sin (p w x)\right) \\
= & \frac{4 \pi\left(1-k^{\prime}\right)}{\left(1-k^{\prime}\right) K(k)} \sum_{n=0} \frac{(-1)^{n} q(k)^{2 n+1}}{1-q(k)^{4 n+2}} \cos ((4 n+2) \pi x / \lambda) \\
= & 1+\sqrt{5-k^{2}}\left(\sum a_{4 p+2} \cos \left(\pi(4 p+2) \frac{x}{\lambda}\right)-\sum a_{4 p} \cos \left(4 \pi n \frac{x}{\lambda}\right)-\sum(-1)^{n} b_{2 p} \sin \left(2 \pi n \frac{x}{\lambda}\right)\right)
\end{aligned}
$$

So $b_{2 n}=0, a_{4 n}=0$ except $a_{0}=\left(5-k^{2}\right)^{-\frac{1}{2}}$ and

$$
a_{4 n+2}=\frac{4 \pi}{K(k) \sqrt{5-k^{2}}} \sum \frac{(-1)^{n} q(k)^{2 n+1}}{1-q(k)^{4 n+2}}
$$

We thus conclude that

$$
\begin{array}{r}
\sqrt{5-k^{2}} \psi(k, x)=1+\frac{2 \pi}{K(k)} \sum\left[2 \frac{(-1)^{n} q(k)^{2 n+1}}{1-q(k)^{4 n+2}} \cos (2 \pi(2 n+1) x / \lambda)+\frac{q(k)^{n+1 / 2}}{1-q(k)^{2 n+1}} \frac{\sin ((2 n+1) \pi x / \lambda))}{\sin \left(n \frac{\pi}{2}-\frac{\pi}{4}\right)}\right] \\
=1+\frac{2 \pi}{K(k)} \sum \frac{q(k)^{n+1 / 2}}{1-q(k)^{2 n+1}}\left[2 \frac{(-1)^{n} q(k)^{n+1 / 2}}{1+q(k)^{2 n+1}} \cos \left((4 n+2) \frac{\pi x}{\lambda}\right)+\frac{\sin ((2 n+1) \pi x / \lambda))}{\sin \left(n \frac{\pi}{2}-\frac{\pi}{4}\right)}\right] \\
\widehat{\Psi}(k, x)=1-\sqrt{5-k^{2}} \psi(k, x)=\frac{k \sqrt{a} S n\left(2 x \frac{K(k)}{\lambda}, k\right)-k^{2} / a}{1-k / \sqrt{a} S n\left(2 x \frac{K(k)}{\lambda}, k\right)}=\frac{k^{\prime}-1+\left(1+k^{\prime}\right) \sqrt{1-k^{\prime}} S n\left(2 x \frac{K(k)}{\lambda}, k\right)}{1-\sqrt{1-k^{\prime}} S n\left(2 x \frac{K(k)}{\lambda}, k\right)} \\
=\frac{2 \pi}{K(k)} \sum \frac{q(k)^{n+1 / 2}}{q(k)^{2 n+1}-1}\left[2 \frac{(-1)^{n} q(k)^{n+1 / 2}}{1+q(k)^{2 n+1}} \cos (2 \pi(2 n+1) x / \lambda)+\frac{\sin ((2 n+1) \pi x / \lambda))}{\sin \left(n \frac{\pi}{2}-\frac{\pi}{4}\right)}\right](30)
\end{array}
$$

and we can now write

$$
\begin{gathered}
\Phi(x, k, \phi)=\frac{K(k)}{\lambda}\left[\widehat{\Psi}\left(k, x-(1-\phi / 2) \frac{\lambda}{2}\right)+\widehat{\Psi}\left(k, x+(1-\phi / 2) \frac{\lambda}{2}\right)\right] \\
=\sum \frac{2 \pi q(k)^{n+1 / 2} / \lambda}{q(k)^{2 n+1}-1}\left[\begin{array}{c}
\left.\frac{2(-1)^{n} q(k)^{n+1 / 2}(\cos (\pi(2 n+1)(2 x / \lambda-(1-\phi / 2)))+\cos (\pi(2 n+1)(2 x / \lambda+(1-\phi / 2))))}{1+q(k)^{2 n+1}}\right] \\
+\frac{\sin \left((2 n+1) \frac{\pi}{2}(2 x / \lambda-(1-\phi / 2))+\sin \left((2 n+1) \frac{\pi}{2}(2 x / \lambda+(1-\phi / 2))\right)\right.}{\sin \left(n \frac{\pi}{2}-\frac{\pi}{4}\right)}
\end{array}\right] \\
=\frac{4 \pi}{\lambda} \sum \frac{(-1)^{n} q(k)^{n+1 / 2}}{1-q(k)^{2 n+1}}\left[2 \frac{q(k)^{n+1 / 2}}{1+q(k)^{2 n+1}} \cos \left((4 n+2) \frac{\pi x}{\lambda}\right) \cos (\pi(2 n+1) \phi / 2)+\frac{\sin ((2 n+1) \pi x / \lambda) \sin \left(\left(n \pi / 2+\frac{\pi}{4}\right) \phi\right)}{\cos \left(n \frac{\pi}{2}+\frac{\pi}{4}\right)}\right]
\end{gathered}
$$


[1] T. Izumitani and T. Hashimoto, J. Chem. Phys., 83, 3694 (1985).

[2] S. Villain-Guillot and C. Josserand, Phys. Rev. E 66, 036308 (2002).

[3] J.S. Langer, Annals of Physics 65, 53 (1971). J.S. Langer, in Solids Far From Equilibrium, edited by C. Godrèche (Cambridge University Press, Cambridge, England 1992), pp. 297-363.

[4] M. Hillert, Acta Met. 9, 525 (1961).

[5] J.W. Cahn and J.E. Hilliard, J. Chem. Phys. 28, 258 (1958).

[6] P.C. Hohenberg and B.I. Halperin, Rev. Mod. Phys. 49, 435 (1977). See also M.C. Cross and P.C. Hohenberg, Rev. Mod. Phys. 65, 851 (1993).For a review, see J.D. Guton, M. San Miguel and P.S. Sahni, in Phase Transition and Critical Phenomena, edited by C. Domb and J.L. Lebowitz (Academic, London, 1983), Vol. 8, p. 267.

[7] C. Chevallard, M. Clerc, P. Coullet and J.M. Gilli, Eur. Phys. J. E 1, 179 (2000).

[8] Y. Oyama, Bull. Inst. Phys. Chem. Res. Rep. 5, 600 (1939). S. Puri and H. Hayakawa, condmat/9901260 and Advances in Complex Systems, Vol. 4, No. 4 (2001) 469-479 .

[9] M.A. Scherer, F. Melo and M. Marder, Phys. Fluids 11, 58 (1999).

[10] A. Stegner and J.E. Wesfreid, Phys. Rev. E 60, R3487 (1999).

[11] J.S. Langer, M. Bar-on and H.D. Miller, Phys. Rev. A, 11, 1417 (1975).

[12] A. Saxena and A.R. Bishop, Phys. Rev. A 44, R2251 (1991).

[13] A. Novik-Cohen and L.A. Segel, Physica D 10, 277 (1984).

[14] M. Abramowitz and I. Stegun, Handbook of Mathematical Functions (Dover, New York, 1965).

[15] F.M. Arscott, Periodic Differential Equations (Pergamon, Oxford, 1981).

[16] $\frac{\partial}{\partial \phi} \Phi(x, k, \phi=0)$ corresponds to a local maximum of the free energy averaged over one period $F(\phi)=\int F(\Phi(x, k, \phi)) d x$. The (G-L) or stationary $(\mathrm{C}-\mathrm{H})$ equation, i.e. the first functional derivative $\frac{\partial F}{\partial \phi}$ admits $\Phi(x, k, \phi=0)=\Psi^{*}(x)$ as solution. But Lamé equation, obtained when linearizing $(\mathrm{G}-\mathrm{L})$ equation around $\Psi^{*}(x)$, is related with the second functional derivative of $F$; therefore one doesn't expect $\frac{\partial}{\partial \phi} \Phi(x, k, \phi=0)$ to be an exact solution or eigenfunction of the Lamé operator $\mathcal{L}$.

[17] P. Coullet, R.E. Goldstein and J.H. Gunaratne, Phys. Rev. Lett J. 63, 1954 (1989).

[18] P.Politi and C. Misbah, Phys. Rev. Lett. 92, 090601 (2004). 


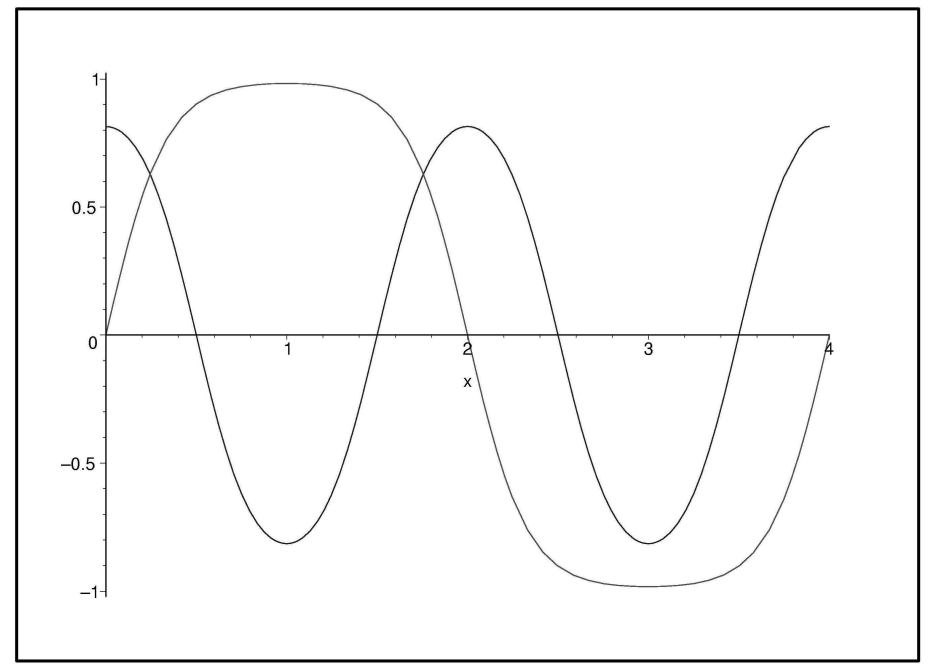

FIG. 1: Profiles of the two first metastable solutions of the $(\mathrm{C}-\mathrm{H})$ dynamics, with $k_{1}^{s}=0.687$ and $k_{2}^{s}=0.985$, corresponding to the first step of the coarsening process. 


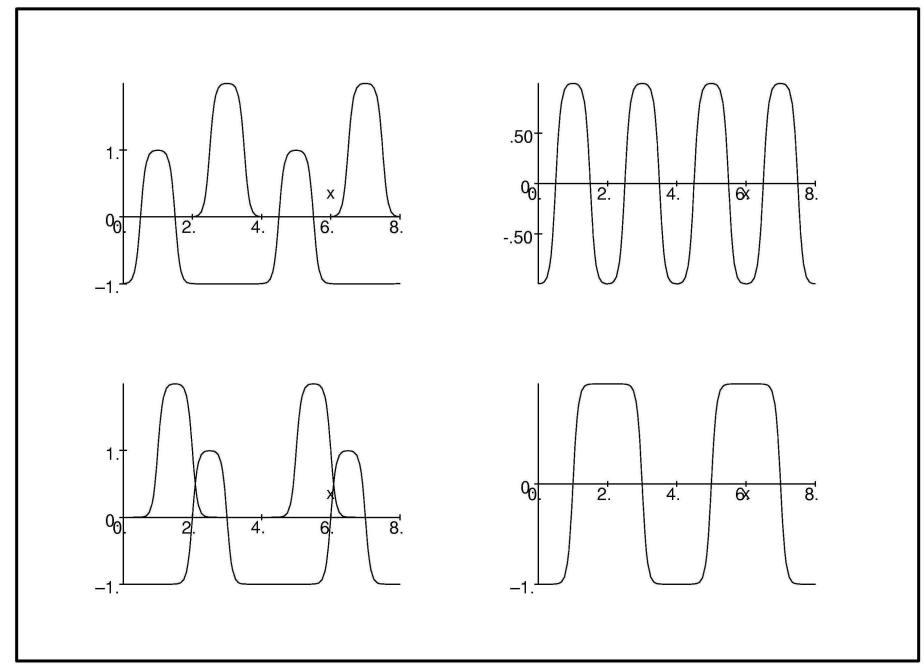

FIG. 2: Construction of the two first steady solutions of the $(\mathrm{C}-\mathrm{H})$ dynamics, with $k_{0}^{s}=0.687$ and $k_{1}^{s}=0.985$, using a superposition of the non-symmetric profile $\psi(k, x)$, itself stationary solution of the $(\mathrm{C}-\mathrm{H})$ equation. By changing the phase shift between the two profiles entering into the linear combination, one obtains two different symmetric profiles, of periods $\lambda$ and $2 \lambda$. 


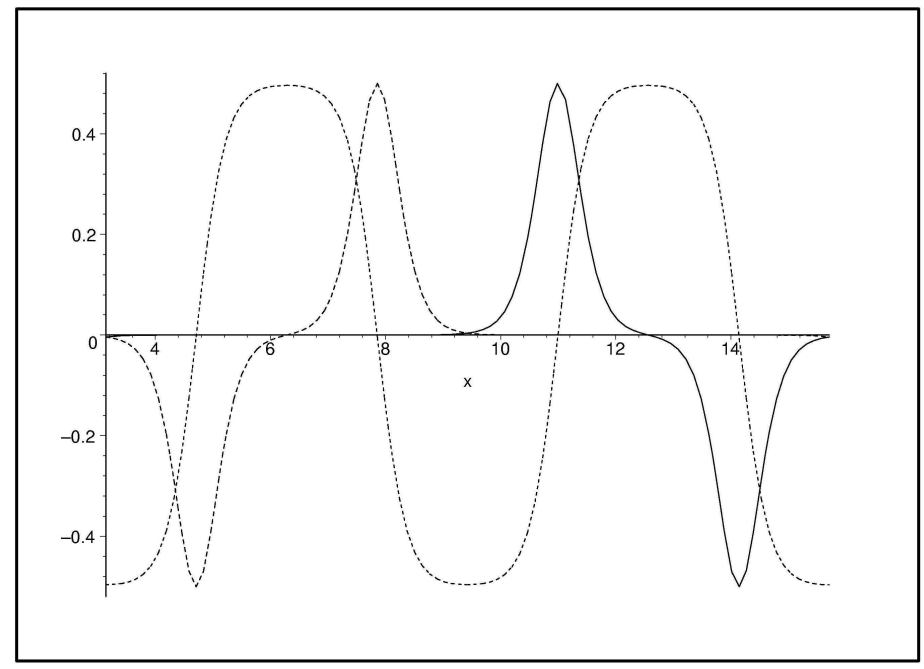

FIG. 3: Langer's most instable perturbation mode of destabilization of the soliton lattice is identified with $\frac{\partial}{\partial \phi} \Phi(x, k, \phi)$ at $\phi=0$. It is composed of two antisymmetric patterns, plotted in dotted (plain) line, evolving toward right (left) at velocity $+\frac{d \phi}{d t}\left(-\frac{d \phi}{d t}\right)$, causing an "antiferro" instability leading to a period doubling of the pattern. They are the spatial derivatives of the initial non symmetric profile $\psi(x)$ which has been used to construct our ansatz in Figure 2. 




FIG. 4: Profile of the free energy landscape during a coarsening process, $\mathcal{F}(\phi)$. It starts at $\phi=0$ for a configuration characterized by the segregation ratio $k_{1}^{s}=0.687$ for which the energy per unit length is $\mathcal{F}(\phi) \simeq-0.135$; one sees that in this region, the free energy is a concave function of $\phi$ and thus, the associated pattern is linearly instable. The elementary step of the coarsening process ends for $\phi=1$ associated with a pattern characterized by the segregation ratio $k_{2}^{s}=0.985$ for which the energy per unit length is $\mathcal{F}(\phi) \simeq-0.45$. In the region $\phi=1$, the free energy is a convex function of $\phi$. 